\title{
The work of a psychosurgical unit
}

\author{
P. K. BRIDGES \\ M.D., Ph.D., M.R.C.Psych. \\ J. R. BARTLETT \\ F.R.C.S.
}

The Geoffrey Knight Psychosurgical Unit, Brook General Hospital, London, SE $184 L W$

\begin{abstract}
Summary
The widespread use of standard leucotomy terminated in the early 1950's with the advent of the phenothiazine group of drugs. But since then, psychosurgical techniques have been considerably refined and the indications for the newer operations are becoming increasingly precise. Knight's (1969) stereotactic tractotomy is probably the most frequently performed contemporary operation for the relief of some psychiatric illnesses, and the number of patients now referred for this treatment have justified the organization of a specific unit for the purpose.

The value of such a special unit, involving close co-operation between neurosurgeon and psychiatrist is emphasiced. It provides a supportive environment for patients who are sharing a highly specialiced treatment, and offers opportunities for studying outcome after operation with resulting improvement in selection. It also facilitates research possibilities in general. The clinical work of the Geoffrey Knight Psychosurgical Unit, probably in its way unique, is described and questions often encountered from doctors and from patients are considered. The importance of rehabilitation after operation is stressed and some aspects of the longer term post-operative psychiatric care involved are dealt with.
\end{abstract}

THE transformation that has taken place in psychosurgical treatment in recent years appears to be unknown to the general public and inadequately understood by many doctors. The popular emotional confusion about contemporary psychosurgery was well characterized in a recent headline-'How 200 mental patients lose part of their brains on the operating table' (Sunday Times, 26 March 1972).

The changes have occurred in two main respects. Firstly, psychosurgical operations have been considerably refined, with the result that no significant effect on the personality is to be expected. Secondly, these operations are now rarely indicated in cases of schizophrenia, for which their value has always been shown to be limited in any case. However, there exists an uninformed yet sophisticated and vocal lay opinion that physical treatments in general, and psychosurgery in particular, are barbaric and irrelevant to psychiatric conditions. Such attitudes uncritically equate physical methods with brutality, and sentimentally assume that psychodynamic methods, desirable as they may be as an ideal and for certain patients, will be of value to all psychiatric cases, without recognizing the considerable and even specific efficacy of physical treatments in particular illnesses.

Modern psychosurgical techniques have been reviewed by Knight (1972). They include the formation of stereotactic (purists may prefer stereotaxic) lesions in the connections of the hippocampus and hypothalamus (Sano et al., 1970), in the cingulum bundle (Ballantine et al., 1967), amygdala (Narabayashi et al., 1963) and corpus callosum (Laitinen, 1972). Some other approaches are described in accompanying papers.

A particular modification, which has been by far the most frequently used clinically, is concerned with the interruption of the connections from the agranular cortex of the cingulate posterior orbital and amygdaloid regions. The lesion is placed beneath the head of the caudate nucleus by the stereotactic insertion of radioactive yttrium seeds $\left({ }^{90} \mathrm{Y}\right)$. The therapeutic effect appears to have specificity in that a generalized reduction in emotional responsiveness is not found.

This technique of subcaudate stereotactic tractotomy, which has also been called pre-innominate (adjacent to the substantia innominata), was developed by Knight (1969), who, in this issue, describes further experiences derived from 660 such operations. The clinical effects have also been reported by Ström-Olsen \& Carlisle (1971) who retrospectively studied 210 patients. They showed that the operation was particularly valuable for cases of depression taken as a group $(56 \%$ recovered or much improved and not requiring treatment) and produced good results in patients with obsessional neurosis $(50 \%)$ and with anxiety states $(41 \%)$. No patient showed major personality changes postoperatively and in only $2.6 \%$ were there moderate or lasting side effects, and these were not socially 
disabling. A subsequent detailed review of the results after operating on patients with obsessional neuroses, compared to the results from a control group with depression has recently been carried out (Bridges, Goktepe \& Maratos, 1973) and in the case of both groups some $70 \%$ recovered or were much improved. Factors of prognostic significance were also discussed.

Over several years, up to his recent retirement, Mr Geoffrey Knight developed a psychosurgical unit at the Brook General Hospital, London, which is in many ways unique. This unit now has twelve beds and about two operations can be carried out each week. Having a group of patients together sharing the common experience of a psychosurgical operation offers a supportive situation and provides especially good opportunities for preparing patients for operation and managing them afterwards. In addition, the concentration of relatively large numbers also aids in the improvement of methods of selection and facilitates research. For all the clinical value of psychosurgery, only a very small proportion of the total number of psychiatric patients will require assessment for operation. Therefore, most psychiatrists will only infrequently encounter a patient who might need this form of treatment and he may well then ask the advice of his own neurosurgical colleague, who is likely himself to see few such cases unless he has a special interest in this type of work. Selection of suitable patients could be less effective in these circumstances and the patient will receive a highly specialized treatment in unsatisfactory isolation. So the value of concentrating psychosurgical work into only a few centres is apparent.

The sources of referral to this unit, some of the complex amalgam of attitudes involved, clinical and personal, and the indications for referral, have been considered recently (Bridges, 1972). The unequivocal indication is that of a depressive illness with symptoms of the endogenous or involutional type of depression, which responds transiently or partially to established treatments and remains chronic or is persistently recurrent. In addition, cases with chronic anxiety, phobic anxiety, tension and obsessional symptoms are likely to do well. The outcome in those with very atypical symptoms will be less certain but psychosurgery may have a special therapeutic place in some of these cases as their response to the more usual treatments is often poor. Schizophrenia is not usually an indication but some cases with prominent depression or tension may be helped. Other forms of psychosurgery may still need to be considered for those rare chronically disturbed schizophrenics who are inadequately controlled by phenothiazines.

Certainly, psychosurgery should only be considered after a full trial of more routine therapies but, in suitable cases, psychosurgical assessment need not be too much delayed, with the patient subjected to poorly controlled symptoms for a prolonged period. Regarding the possibility of psychosurgery only as a final desperate measure is an undesirable clinical approach in relation to contemporary operations, and it can affect the result adversely as mentioned later.

The present report deals with some of the practical aspects of referral and describes the general function of the unit, which is part of the South East Metropolitan Regional Neurosurgical Centre. For reasons of the availability of accommodation, most of the beds form part of a medical ward largely of singlebedded rooms, with which it shares nurses. One of the staff nurses of this ward is appointed with psychiatric experience but the others are general nurses. Eight beds form one wing of the ward and somemore bedsarealso available in a small peripheral hospital where patients can be transferred as they progress, and where they are expected to look after themselves with minimal nursing and domestic assistance. The ward has a pleasant day-room where all patients spend the day and take their meals. Throughout their admission the patients are up and about normally except for about two days at the time of operation.

Patients come from all parts of the country and most are accepted for assessment from psychiatrists but occasionally referrals may come directly from family doctors in those unusual circumstances where the patient is not receiving psychiatric care. All first attend the unit as an out-patient for a neurosurgical and psychiatric assessment, except for those who live a long distance away who may be admitted for a short time for assessment. Out-patients wait with the in-patients in the day room so that some exchange of opinion can take place and the referred patients can discuss the operation with those who have undergone the experience. Patients need not be fully committed to the possibility of operation at the time of assessment and the situation can be discussed with those who are undecided, arrangements being made for a second consultation later where appropriate.

A close relative is always asked to attend with the patient being assessed so that they can give additional information and discuss the implications of operation with the social worker attached to the unit. She is also able to give support to the relatives during the patient's admission and in the period after discharge when social problems may arise with which the social worker can help in association with the patient's local social services.

No particular formality is required for the initial referral except the psychiatrist's opinion with a psychiatric and medical history, preferably with 
previous hospital notes. The staff of the unit includes a clinical psychologist and research is in progress attempting to evaluate various psychological instruments in the assessment of patients. On clinical grounds it seems that, unlike the leucotomies, in the case of subcaudate tractotomy, poor personality is not necessarily a contra-indication and neither is the presence of a current adverse social situation. Suitable patients incapacitated by affective illnesses, with vulnerable personalities, show improvement in personality function when relieved of abnormally excessive emotional reactions by operation, although post-operative support will be particularly important in these cases. Two patients recently were operated on, one just before and the other after the death of a spouse. In each case severe depression had occurred during previous crises and it was hoped that the patient would be relieved of an overwhelming psychiatric illness when deprived of the support of their partner, and this proved to be so in these cases. However, the clinical prominence of an inadequate personality and of inability to manage social stress often suggests an essentially neurotic type of illness which is less likely to be helped by operation. Advanced age is not a contra-indication and elderly patients frequently do well.

Of course, every surgical effort is made to produce a minimal operation scar. In most cases, because the hairline tends to recede and in order to faciliate the surgical approach, a horizontal incision is made across the forehead through a skin crease. For some women a coronal incision is made just behind the hairline over the head. Most scars are virtually invisible after a few months. From the psychiatric point of view the scar has been regarded as of special importance to the patient and this matter is always raised for discussion, but in practice it is an aspect which has proved to be of rather less concern to most patients than expected. It seems that when the patient has made the difficult decision to agree to operation, perhaps after a long period of illness, the scar is of relatively less significance. However, a detectable scar can lead to social problems. Bearing in mind the considerable and unreasonable prejudice about psychosurgery, the possibility that a patient has had such an operation can produce interpersonal difficulties and sometimes almost insuperable problems in relation to work. Many people, employers of course included, still quite erroneously believe that a psychosurgical operation implies that the patient has been a severely disturbed schizophrenic who has probably suffered personality damage postoperatively, all of which is most unlikely in the context of contemporary psychosurgery.

Patients are usually admitted from the waiting list about 1 week before operation, which gives time for them to settle into the ward, allowing observation and a detailed clinical assessment to be carried out. In addition, many patients are on high doses of medication, and sometimes a wide variety. This is reduced in this pre-operative period when simple sedation with diazepam (Valium) is all that is needed and phenothiazines, in particular, are undesirable. It is perhaps unnecessary to stress that patients must stop taking monoamine oxidase inhibitors at least 2 weeks before admission. Especially disturbed or distressed patients may be admitted only a few days before operation or even the day before. All operations are carried out with a general anaesthetic and the head is not shaved; points often asked about by patients.

Patients are up $24 \mathrm{hr}$ after operation, often with some headache and perhaps nausea for a few days, which is treated if necessary with codeine compound tablets and perphenazine (Fentazin). All other medicaton is stopped after operation, allowing observation of the patients's response, and the continuation of medication without reappraisal of its need is avoided. However, later on, further medication may be indicated and there is some clinical evidence that routine treatments, including electroplexy, can prove more effective after operation than before. Anti-convulsant medication is not required routinely after operation.

The need for ancillary treatments in some cases after operation has been stressed previously (Bridges, 1972). In particular the abnormal mood state contributing to phobic anxiety may well be improved but conditioned anxiety often needs behaviour therapy post-operatively, especially if it has been present for some time. Methods described by Gaind, Watson $\&$ Marks (1971) and carried out by $\mathrm{Dr}$ Gaind on some of our phobic patients, have been found of considerable value in these cases. Indeed, one of the indications for psychosurgery is severe phobic anxiety that has failed to respond to routine treatments, including de-conditioning, and it has been found that such cases are then more likely to respond to similar behaviour therapy after operation. Similarly, subsequent treatment for residual obsessional symptoms as described by Levy \& Meyer (1971) has been used with success. Other aspects of post-operative rehabilitation are considered later.

It used to be our practice to perform an air encephalogram prior to operation but experience of a large number of cases showed that this produced little useful information with regard to the preoperative assessment of the case. We now insert a small amount of air into the right lateral ventricle at the time of operation in order to demonstrate the anterior horns of the lateral ventricular system and also the anterior end of the third ventricle. These observations may modify the calculations involved in siting the lesion. The operation itself appears to 
produce almost no distress and the principal discomfort is occasioned by preliminary anticipation which is ameliorated as far as possible by support and medication.

Improvement after stereotactic tractotomy is usually curiously delayed. It is rare for patients to recover almost immediately after operation, although a few do, but the majority seem to take an average of 3-6 months before improvement becomes clearly apparent and, especially those who have been severely ill for a long period, full recovery may take up to a year or even more. The delay is stressed to all patients, who otherwise tend to expect dramatic results in a short time from a surgical operation, and in this way disappointment is avoided. A final, reliable assessment of the result cannot be made in under 2 years.

Some patients experience lethargy soon after the operation and they need stimulation to facilitate the regaining of their energy and interest. This activation is carried out by the encouragement of the ward nurses and by the unit's occupational therapist. The problem of lethargy is usually overcome in about a week but in some it may last longer and stimulation has then to be maintained. Some patients become confused for a time post-operatively. This appears to be an age-related side effect as it is almost unknown in those under 50 years while it is to be expected temporarily in some degree in most elderly patients.

There is a weekly group meeting of the patients, which gives opportunities for interaction between patients before operation and those in the postoperative period. Patients usually stay for about 4-8 weeks and during this time progressive activity, including outings, is encouraged. Most can then be discharged home but some require further postoperative rehabilitation and return to the referring hospital. Those who live a long distance away, and so tend to miss their families, may return to their local hospital sooner, perhaps after 2 weeks.

The importance of rehabilitation cannot be overemphasized and this need does not appear to be always fully understood. Active rehabilitation is necessary in order to overcome post-operative lethargy in the short term and to help the patient realize new potentials in the longer term. Institutional or clinical dependence and the acceptance of illness can become a way of life in chronically disabled patients and, while their tendency to experience depression or anxiety may be considerably reduced by psychosurgery, a new independent and more confident existence has to be developed, which will be a slow process in those who have had long illnesses. Furthermore, the gradual improvement that occurs after psychosurgery requires a similar gradual pace of rehabilitation.
Psychosurgery offers renewed therapeutic endeavour which begins with operation, but if the treatment is seen as a last, hopeless resort, it can prove to be so for that reason alone. One lady, who had been in hospital for many years with chronic depression, was transferred back to the referring hospital after operation. She was re-admitted to the long-stay ward where she had previously been for a long period and there was no change in treatment or clinical approach. The patient was told that she had received the ultimate therapy and if she did not improve nothing further could be done. But no effort was made to facilitate any post-operative improvement there may have been. She therefore later discharged herself, as was found at routine follow-up, when she said she was no better. Except for the finding that she was out of hospital, we are unsure whether the operation had helped this lady or not because of the absence of post-operative help; and the obviously pessimistic view of the psychiatric department looking after her was simply confirmed by their therapeutic withdrawal from the patient after the possibility of improvement had been presented by operation.

Patients are followed up routinely 6 months after operation and then 6 months later. A second operation is helpful in some cases, although these constitute only a small group and need to be especially carefully selected. This decision is unlikely to be reliable before about 1 year after the first attempt. $A$ stereotactic tractotomy in the cingulate region is the usual second operation, and appears to be useful when depression has responded to the subcaudate operation, but tension, anxiety or obsessional symptoms persist and do not respond to routine treatments. Occasionally the previous lesion may be extended by the insertion of further seeds into the subcaudate region if the original placement subsequently appears to be less than optimal.

Follow-up studies are continuing and it is becoming increasingly apparent that the research need is now not so much to show in how many selected cases the operation significantly relieves symptoms, which has been the approach in previous investigations, but more for methods of identifying those cases which are likely to respond. Selection must at the moment depend upon clinical observation, but the assessment of cases by means of psychological and biochemical investigations is underway, which may produce additional more objective information (Bridges, 1973).

Psychosurgery is relevant to only a very small proportion of the total psychiatric population and, with the increasing efficacy of other treatments, particularly for depression, the aim remains to relieve suffering while producing minimal side effects, if any, until psychiatric therapy reaches a stage where surgi- 
cal intervention becomes unnecessary. In the meantime it is becoming clear that there is a definite clinical place for selective psychosurgery, the challenge being to precisely locate the indications within the total field of psychiatry. There is a further and peripheral speculation that, with contemporary preoccupation with cost-effectiveness, the relatively high expense involved in a psychosurgery unit is likely to be more than repaid by reducing the need for psychiatric admission and ongoing psychiatric care, an aspect clearly shown in Tables 8 and 9 in the study by Bridges, Goktepe \& Maratos (1973).

\section{Acknowledgments}

We are grateful to $\mathrm{Mr}$ Geoffrey Knight for help in the preparation of this paper and we acknowledge the support of the South East Metropolitan Regional Hospital Board for research in the unit.

\section{References}

Ballantine, H.T., Cassidy, W.L., Flanagan, N.B. \& MARINO, R. (1967) Stereotaxic anterior cingulotomy for neuropsychiatric illness and intractable pain. Journal of Neurosurgery, 26, 488.

Bridges, P.K. (1972) Psychosurgery today; psychiatric aspects. Proceedings of the Royal Society of Medicine, 65, 1104.
BRIDGES, P.K. (1973) Methods of assessing patients for psychosurgery and their outcome after operation. Psychiatria, Neurologia et Neurochirurgia, 76, 335.

Bridges, P.K., GoktePe, E.O. \& Maratos, J. (1973) A comparative review of patients with obsessional neurosis and with depression treated by psychosurgery. British Journal of Psychiatry 123, (Dec).

Fulton, J.F. \& JACOBSEN, C.F. (1935) Abstracts of the Second International Neurological Congress, London.

GaInd, R., Watson, J.P. \& Marks, I.M. (1971) Some approaches to the treatment of phobic disorders. Proceedings of the Royal Society of Medicine, 64, 1118.

KNIGHT, G.C. (1969) Bi-frontal stereotactic tractotomy. British Journal of Psychiatry, 115, 257.

KNight, G.C. (1972) Psychosurgery today: neurosurgical aspects. Proceedings of the Royal Society of Medicine, 65, 1099.

LAITINEN, L.V. (1972) Stereotactic lesions in the knee of the corpus callosum in the treatment of emotional disorders, Lancet, i, 472.

LEVY, R. \& MEYER, V. (1971) Ritual prevention in obsessional patients. Proceedings of the Royal Society of Medicine, 64, 1115.

Narabayashi, A., Nago, T., Saito, Y., Yoshida, M. \& NaGAHATA, M. (1963) Stereotaxic amygdalotomy for behaviour disorders. Archives of Neurology, 9, 1.

Sano, K., Mayanagi, Y., Sekino, H., Ogashiwa, M. \& IsHiJima, B. (1970) Results of stimulation and destruction of the posterior hypothalamus in man. Journal of Neurosurgery, 33, 689.

Ström-Olsen, R. \& Carlisle, S. (1971) Bi-frontal stereotactic tractotomy. British Journal of Psychiatry, 118, 141. 